\title{
Is the Band a Good Procedure Choice, and for Whom Should We Still Consider It?
}

\author{
Patrick Hartendorp • Dana A. Telem
}

Published online: 5 July 2013

(c) Springer Science + Business Media New York 2013

\begin{abstract}
Bariatric surgery remains the only durable weight loss treatment in the morbidly obese patient. Since 1993, laparoscopic adjustable gastric banding has been a popular choice owing to low perioperative morbidity and relative ease of placement. LAGB functions by limiting food intake via placement of an inflatable silicone band around the stomach. Although LAGB may provide significant reductions in weight and comorbidity resolution, it is not the most effective surgical procedure. In addition, the rate of reoperation due to late complications and failure has become a growing concern. These issues have caused some surgeons to question the role of banding altogether.
\end{abstract}

Keywords Obesity - Bariatric surgery - Adjustable gastric band · Outcomes · Weight loss

\section{Introduction: Problem of Obesity}

Obesity has emerged as one of the most important public health issues in the USA and globally. Typically, obesity is defined as a body mass index (BMI) of $30 \mathrm{~kg} / \mathrm{m}^{2}$ or greater, and can be further divided into four subclasses: obesity $\left(30-34.9 \mathrm{~kg} / \mathrm{m}^{2}\right)$, moderate obesity $\left(35-39.9 \mathrm{~kg} / \mathrm{m}^{2}\right)$, morbid obesity $\left(40-49.9 \mathrm{~kg} / \mathrm{m}^{2}\right)$, and supermorbid obesity $\left(50 \mathrm{~kg} / \mathrm{m}^{2}\right.$ or greater). The consequences of morbid obesity may include premature death and an increased risk of

P. Hartendorp · D. A. Telem ( $₫)$

Bariatric and Metabolic Weight Loss Center, Division of General Surgery, Stony Brook University Medical Center, 100 Nicholls Road, HSC T18-040, Stony Brook, NY 11794, USA

e-mail: dana.telem@stonybrookmedicine.edu weight-related health problems, including, but not limited to, diabetes, hypertension, hypercholesterolemia, heart disease, osteoarthritis, sleep apnea, and gallbladder disease (Table 1). With the prevalence of obesity rising in both developed and developing nations, increased efforts and costs have been directed toward prevention and treatment. In the USA, it is estimated that up to $33 \%$ of men and $36 \%$ of women are considered obese, resulting in health care expenditure of approximately $\$ 100$ billion per year to treat obesity and its complications $[1,2 \bullet]$. Current treatment options include both medical and operative options. Medical management centers on dietary and behavioral modifications, exercise regimens, and pharmacologic agents when appropriate [3]. Unfortunately, medical management alone is rarely successful in achieving and maintaining significant weight loss in the obese population. For morbidly obese patients with a significant amount of weight to lose, bariatric surgery remains the only durable method of sustained weight loss [4]. In this article, we will discuss gastric banding, a restrictive procedure developed in the 1970s as a surgical treatment for obesity.

\section{Brief History of Gastric Banding}

Beginning in the 1970s, surgeons sought to develop approaches to decrease gastric capacity while maintaining gastrointestinal continuity. In 1978, the first nonadjustable gastric band was credited to two surgeons from New Mexico, Lawrence H. Wilkinson and Ole A. Peloso [5]. Their initial procedure involved inversion of the lesser curvature of the stomach by performing a Nissen fundoplication followed by gastric wrapping with mesh [5]. Other researchers performed similar operations. In 1982, Kolle in Norway described placement of a nonadjustable 
Table 1 Obesity-related comorbidities

\begin{tabular}{ll}
\hline Type 2 diabetes & Asthma \\
Hyperlipidemia: & Cancer \\
Hypercholesterolemia & Breast, endometrium \\
Hypertriglyceridemia & Colon, prostate \\
Coronary artery disease & Depression \\
Hypertension & Sex hormone anomalies \\
Obstructive sleep apnea & Stress urinary incontinence \\
Obesity hypoventilation syndrome & Venous stasis disease \\
Degenerative joint disease & Steatohepatitis \\
\hline
\end{tabular}

gastric band during an open surgery. In the following year, Molina and Oria in Texas reported a gastric segmentation procedure using a nonadjustable Dacron graft to wrap the proximal stomach. Similar procedures were performed by Naslund in Sweden and Frydenburg in Australia using Marlex mesh and silicone, respectively. US researchers Bashour and Hill experimented with a "gastro-clip gastroplasty" in which a polypropylene clip with steel reinforcements and a fixed stoma was clasped horizontally at the gastric cardia, creating a proximal pouch [6]. These early bands, grafts, and clips were unsuccessful, with a high failure rate. They did not establish an ideal stoma diameter and often led to prolapse of the stomach upward through the band (slippage) [6].

Austrian surgeons Szinicz and Schnapka were the first to bring the concept of "adjustability" to gastric banding. They experimented with a silicone elastomer ring which contained a balloon attached to a subcutaneous port. The volume of the band balloon could be adjusted by adding or removing saline via the port. This concept was further cultivated by Dag Hallberg and Peter Forsell, in Sweden,who described what is now known as the Swedish adjustable gastric band in 1985. In parallel, Lubomyr Kuzmak, a Ukrainian surgeon, reported on the clinical use of an inflatable Silastic band. Both of these bands connected to a subcutaneous port through which fluid could be added or removed to modify the degree of constriction. In 1986, Kuzmak reported improved weight loss and decreased complications in comparison with the nonadjustable band he had been using since 1983 [6].

In the USA, two FDA-approved laparoscopic implantable gastric banding devices (LAP-BAND from Allergan and REALIZE adjustable gastric band from Ethicon EndoSurgery) are currently available [7]. The differences between the two devices include band shape, band size, and port size. The LAP-BAND system offers two sizes of concentric bands, the AP standard (10-ml fill) and AP large (14-ml fill), paired with a 11.9- or 14.7-mm access port. The REALIZE Band-C is available as a one-size band with a low-profile 11.6-mm access port [8].

\section{Gastric Banding: Indications and Mechanisms of Weight Loss}

The four commonest bariatric procedures are the Rouxen-Y gastric bypass, the adjustable gastric band, the duodenal switch with biliopancreatic diversion, and vertical sleeve gastrectomy. These operations achieve weight loss via restriction of caloric intake (gastric band and sleeve gastrectomy), or a combination of restriction and intestinal malabsorption of calories (gastric bypass and duodenal switch) plus some metabolic components [4].

The indications for the aforementioned weight loss operations were outlined in 1991 by the National Institutes of Health [4]. Patients are currently considered candidates for bariatric surgery if they have a BMI of $40 \mathrm{~kg} / \mathrm{mg}$ [2•] or greater, or a BMI greater than $35 \mathrm{~kg} / \mathrm{mg}^{2}$ with an obesityrelated comorbid condition. Obesity-related comorbidities include diabetes, hypertension, sleep apnea, and functional impairments limiting employment or activities of daily living (Table 1). In 2011, however, the FDA expanded the use of the LAP-BAND only to include obese individuals with a BMI of $30-34 \mathrm{~kg} / \mathrm{mg}^{2}$ who also have an existing condition related to their obesity or whose BMI is greater than $35 \mathrm{~kg} / \mathrm{mg}^{2}$ regardless of comorbidity. Potential candidates must also demonstrate prior unsuccessful attempts at medically supervised weight reduction. Relative contraindications include the inability to comply with postoperative requirements and follow-up, active alcohol or substance abuse, and uncontrolled psychiatric disease [4].

Gastric banding functions by limiting food intake via placement of an inflatable silicone band around the stomach below the gastroesophageal junction. The band is attached by tubing to a port with is implanted subcutaneously and allows adjustment of the size of the outlet via the addition or removal of saline [3] (Fig. 1). The position of the band creates a small pouch at the top of the stomach which holds approximately $4 \mathrm{oz}$, whereas the typical stomach possesses a 48-oz capacity. The gastric band slows the passage of food from the pouch to the lower part of the stomach [9]. It is postulated that as the proximal part of the stomach registers as full, peptide tyrosine tyrosine is secreted from gastrointestinal mucosa, signaling the sensation of satiety. Thus, patients with gastric bands are hungry less often, experience early satiety, eat smaller portions, and lose weight over time $[7,9,10]$.

\section{Laparoscopic Placement and Postoperative Care}

The emergence of laparoscopy in the 1990s led to tremendous progress in the field of bariatric surgery and an increase in the appeal of the gastric band for the management of obesity. In 1993, the first laparoscopic human 

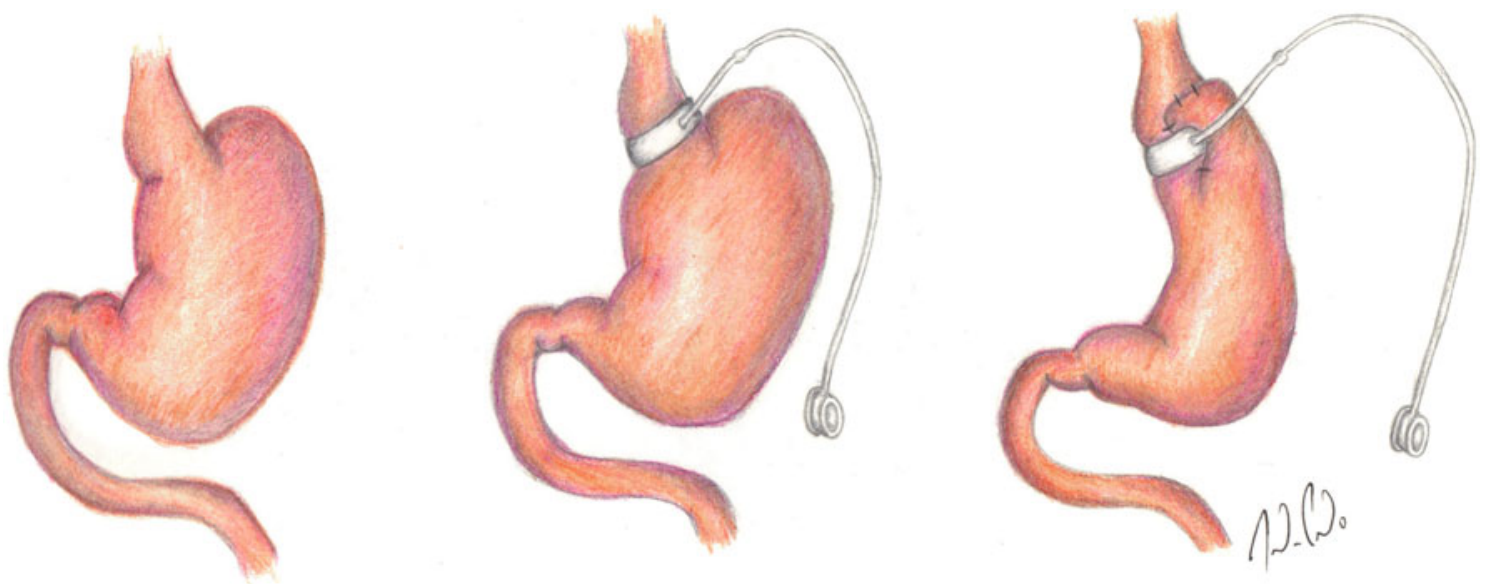

Fig. 1 Placement of the adjustable gastric band. (Courtesy of Andrew Peredo)

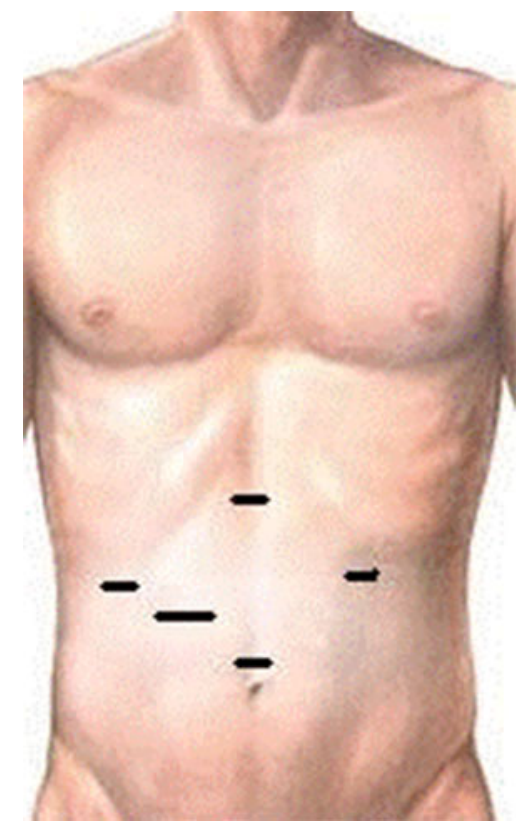

Fig. 2 Trocar placement for laparoscopic adjustable gastric banding. Position 4 for a $15-\mathrm{mm}$ trocar to facilitate band placement. All other positions indicate $5-\mathrm{mm}$ trocars

implantation of the adjustable gastric band was performed by Belachew and Le Grand in Belgium. This paved the way for laparoscopy being the preferred method of implantation [6] (Fig. 2). Surgeons continue to develop laparoscopic innovations involving the band, including implantation via single-port laparoscopy [9].

The laparoscopic adjustable gastric banding (LAGB) procedure offers several operative advantages, including technical ease of placement, quick recovery time, and potential reversibility of the procedure. The disadvantages include the requirement for serial adjustments after surgery, and success often depends on patient compliance and close follow-up [4]. Laparoscopic adjustable gastric band adjustment frequency guidelines differ between manufacturers but are tailored to a weight-loss goal of 1-2 lb/week [11]. Some manufacturers promote an average of four adjustments during the first year, two during the second year, and an additional two during the third year after surgery [11]. Although studies regarding the frequency of adjustments are limited, initial reports suggest more frequent adjustments may result in increased excess weight loss (EWL) after LAGB. The optimal frequency of band adjustments remains unknown [11].

In our practice, LAGB is performed as an outpatient procedure or may require a one-night hospital stay depending on patient characteristics and comorbidities. Patients initially receive a clear liquid diet following surgery, and routine postoperative imaging and laboratory work are not done unless they are clinically indicated. The first gastric band adjustment typically occurs 4-6 weeks following the operation, and patients are seen in the office every 4-6 weeks for subsequent adjustments until the "green zone" is obtained. The "green zone" is defined as the appropriate amount of fluid which affords satiety and weight loss, without symptoms or regurgitation, reflux, or discomfort.

\section{Outcomes of LAGB}

As FDA approval of the band was not received until 2001, most of the early and long-term literature regarding LAGB comes from Europe and Australia, where surgical experience extends back to 1993 [12]. There is a trend in the recent literature to compare LAGB and laparoscopic Rouxen-Y gastric bypass (RYGB), the two commonest bariatric procedures. In this section, we examine perioperative morbidity and mortality, efficacy in terms of weight loss and resolution of comorbidities, and complications of 
LAGB in comparison with other bariatric procedures, mainly RYGB.

Perioperative Morbidity and Mortality

By requiring no bowel transection and maintaining the body's natural gastrointestinal continuity, LAGB lends itself to lower perioperative morbidity and mortality [12]. Mortality rates on the order of $1: 1,000$ to $1: 3,000$ are commonly reported [12]. In one 15 -year follow-up study of 3,227 patients who underwent LAGB, there were no deaths associated with primary laparoscopic adjustable gastric band placement or any subsequent revisional procedures [13••]. A meta-analysis demonstrated that although mortality was low overall, fewer deaths occurred in patients undergoing LAGB versus RYGB $(0.06 \%$ vs $0.17 \%$, respectively) [3].

In terms of perioperative morbidity, several US studies have demonstrated that LAGB is safer than other common bariatric procedures in the early postoperative period [12]. The risks of short-term complications such as perforation, venous thromboembolism, hemorrhage, infection, and leak are significantly decreased for LAGB versus RYGB [3]. In addition, operative time and hospital length of stay are significantly decreased for LAGB as compared with other bariatric operations [14]. LAGB operative times were shorter by a median of $68 \mathrm{~min}$, and the hospital length of stay was approximately 2 days shorter [3].

\section{Weight Loss}

In a systematic review by Tice et al. [3] of 12 studies comparing LAGB versus RYGB, the percentage EWL at 1 year after LAGB surgery ranged from 33 to $54 \%$. The median difference in percentage EWL between LAGB and RYGB at 1 year was $25 \%$ in favor of RYGB [3] (Table 2). Further studies have demonstrated that the pattern of weight loss after LAGB is not as rapid as that after RYGB and sleeve gastrectomy. Weight loss from LAGB progresses over 2 years and then plateaus [12] (Figure 3). At 5 years after surgery, the mean percentage EWL is estimated to be $30-55 \%$ [12]. Although weight loss is slower than that from other bariatric procedures, several studies have demonstrated long-term sustainable weight loss of up to $50 \%$ EWL after LAGB [12]. The notion that weight loss following LAGB is sustainable is further supported by a 15-year follow-up of LAGB patients conducted by O'Brien et al. [13••]. The results from this study demonstrated $47.1 \%$ EWL at 15 years after LAGB, with mean $47 \%$ EWL for all patients at or beyond 10 years' follow-up [13••]. This study also observed that the weighted mean percentage EWL for LAGB is comparable to that of RYGB and gastroplasty (Table 3).
Table 2 Percentage excess body weight loss after 1 year

\begin{tabular}{lll}
\hline Study & LAGB $(n)$ & RYGB $(n)$ \\
\hline Biertho 2003 & $33(805)$ & $67(456)$ \\
Weber 2004 & $35(103)$ & $55(103)$ \\
Jan 2005 & $34(154)$ & $64(219)$ \\
Mognol 2005 & $41(179)$ & $63(111)$ \\
Parikh 2005 & $35(197)$ & $58(97)$ \\
Bowne 2006 & $31(60)$ & $52(46)$ \\
Cottam 2006 & $48(181)$ & $76(181)$ \\
Galvani 2006 & $39(470)$ & $65(120)$ \\
Kim 2006 & $34(160)$ & $64(232)$ \\
Rosenthal 2006 & $54(152)$ & $73(849)$ \\
Angrisani 2007 & $35(27)$ & $51(24)$ \\
Jan 2007 & $34(406)$ & $65(492)$ \\
\hline
\end{tabular}

Data from Tice et al. [3]

$L A G B$ laparoscopic adjustable gastric banding, RYGB Roux-en-Y gastric bypass

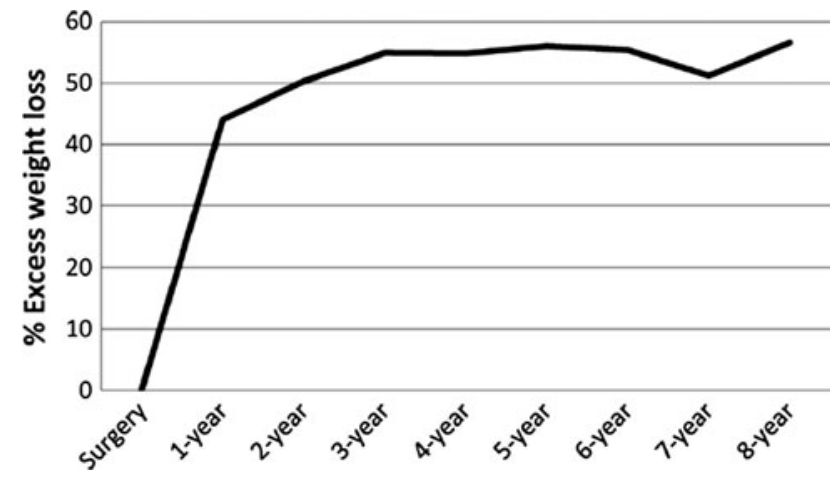

Fig. 3 Mean excess weight loss at each year of 25 observational studies reporting weight results at more than 5 years. Studies included 12,129 people at the baseline. The percentage excess weight loss is calculated from the weight at a body mass index of $25 \mathrm{~kg} / \mathrm{m}^{2}$. Included are all studies published in the English literature with more than 5-year weight loss expressed as percentage excess weight loss before July 1, 2010. (Reprinted from Dixon et al. [12]; copyright 2012, Lippincott, Williams \& Wilkins/American Heart Association, with permission)

Table 3 Percentage excess weight loss $(\% E W L)$ with ranges for bariatric procedures

\begin{tabular}{llc}
\hline Procedure & Weighted mean \%EWL & Range of \%EWL \\
\hline RYGB & 54.0 & $28-68$ \\
LAGB & 54.2 & $33-64$ \\
Gastroplasty & 52.9 & $-10-62$ \\
BPD/DS & 73.3 & $70-75$ \\
\hline
\end{tabular}

Data from Tice et al. [3]. Pooled data from systematic review [16] with a weighted mean \%EWL for the principal procedures.

$B P D / D S$ biliopancreatic diversion with duodenal switch 
Impact on Weight-Related Comorbidities

The positive impact of sustained weight loss on obesityrelated comorbidities is well documented [12]. Weight loss after LAGB is accompanied by improvements in or normalization of insulin sensitivity and glycemia, obesityrelated dyslipidemia, C-reactive protein and other proinflammatory cytokine levels, nonalcoholic fatty liver disease, sleep disturbance, and ovulatory function and fertility in women with polycystic ovary syndrome [12]. After a median follow-up of 13 months, Brancatisano et al. [15] observed resolution and or improvement of comorbidities as follows: type 2 diabetes mellitus, $79 \%$; metabolic syndrome, $78 \%$; hypertension, $67 \%$; dyslipidemia, $66 \%$; gastroesophageal reflux, $66 \%$; asthma, $57 \%$; arthritis, $70 \%$; polycystic ovary syndrome, $48 \%$; and depression, $57 \%$. Resolution of obesity-related comorbidities at 1 year after LABG and RYGB has also been compared (Table 4). Although both procedures proved to be beneficial, RYGB resulted in greater percentage resolution for almost all comorbidities. This finding is likely attributed to the increased percentage EWL associated with RYGB as well as the metabolic component of the procedure [3].

\section{Late Morbidity}

Although early complications of LAGB are typically secondary to technical issues associated with band placement, port site, or wound issues, patients who undergo LAGB are predisposed to developing unique "late" band-related complications. Such complications include band slippage, band deflation from a ruptured balloon or inflation port breakage, infection of the band or subcutaneous port, and band erosion through the gastric lumen [16] (Table 5). It is estimated that up to $33 \%$ of patients who undergo LAGB will require a reoperation or revision to another bariatric procedure either secondary to band-specific complications or inadequate weight loss $[12,16,17]$.

Late problems after band placement have differed greatly in incidence since the introduction of LAGB in 1993 [12]. There has been a general reduction in the incidence of the common reasons for revisional LAGB surgery related to improvement in placement technique, technical changes in the bands and adjustment systems, and

Table 5 Commonest complications after LAGB and RYGB

\begin{tabular}{|c|c|c|}
\hline & LAGB & RYGB \\
\hline \multirow[t]{5}{*}{ Early } & Gastric perforation & Staple line bleed \\
\hline & Band or port site infection & Anastomotic leak or bleed \\
\hline & Band obstruction & $\begin{array}{l}\text { Bowel injury or } \\
\text { obstruction }\end{array}$ \\
\hline & Malposition & Pouch dilation \\
\hline & $\begin{array}{l}\text { Stoma obstruction with } \\
\text { perforation }\end{array}$ & \\
\hline \multirow[t]{6}{*}{ Late } & $\begin{array}{l}\text { Acute gastric prolapse/ } \\
\text { band slippage }\end{array}$ & Internal hernia \\
\hline & $\begin{array}{l}\text { Band erosion, } \\
\text { dysfunction, leak }\end{array}$ & $\begin{array}{l}\text { Adhesive bowel } \\
\text { obstruction }\end{array}$ \\
\hline & $\begin{array}{l}\text { Chronic band slippage/ } \\
\text { pouch dilation }\end{array}$ & $\begin{array}{l}\text { Anastomotic stenosis, } \\
\text { leak, or fistula }\end{array}$ \\
\hline & $\begin{array}{l}\text { Port rotation or leak, skin } \\
\text { irritation }\end{array}$ & $\begin{array}{l}\text { Marginal ulcer/gastritis } \\
\text { and stenosis }\end{array}$ \\
\hline & Esophageal dilation & Incisional hernia \\
\hline & Explantation & Nutrient deficiency \\
\hline $\begin{array}{l}\text { Common to } \\
\text { both }\end{array}$ & \multicolumn{2}{|c|}{$\begin{array}{l}\text { Wound infection, incisional hernia, symptomatic } \\
\text { cholelithiasis, nausea/vomiting, intra-abdominal } \\
\text { bleeding, pulmonary embolism }\end{array}$} \\
\hline
\end{tabular}

Table 4 Percentage resolution of comorbidities

\begin{tabular}{|c|c|c|c|c|c|c|c|c|}
\hline Study & Arm & DM & HTN & Dyslipidemia & OSA & GERD & Arthritis & Asthma \\
\hline \multirow[t]{2}{*}{ Weber 2004} & LAGB & 59 & 70 & 0 & - & - & - & - \\
\hline & RYGB & 84 & 75 & 50 & - & - & - & - \\
\hline \multirow[t]{2}{*}{ Bowen 2006} & LAGB & 40 & 27 & 40 & 34 & - & 14 & 12 \\
\hline & RYGB & 100 & 63 & 43 & 88 & - & 29 & 29 \\
\hline \multirow[t]{2}{*}{ Cottam 2006} & LAGB & 50 & 56 & 46 & - & - & - & - \\
\hline & RYGB & 78 & 81 & 81 & - & - & - & - \\
\hline \multirow[t]{2}{*}{ Galvani 2006} & LAGB & 68 & 59 & - & 55 & 56 & 60 & - \\
\hline & RYGB & 75 & 61 & - & 63 & 75 & 69 & - \\
\hline \multirow[t]{2}{*}{ Kim 2006} & LAGB & 77 & 56 & 37 & - & 88 & 84 & - \\
\hline & RYGB & 72 & 66 & 48 & - & 84 & 75 & - \\
\hline \multirow[t]{2}{*}{ Angrisani 2007} & LAGB & - & 0 & - & 100 & - & - & - \\
\hline & RYGB & 100 & 0 & 100 & - & - & - & \\
\hline
\end{tabular}

Data from Tice et al. [3]

$D M$ diabetes mellitus, GERD gastroesophageal reflux disease, HTN hypertension, OSA obstructive sleep apnea 
less aggressive use of restriction [12]. Among LAGB patients over the last 15 years, O'Brien et al. [13••] found a revision rate of approximately $40 \%$ in the first 10 years, which decreased over time with experience to $6.4 \%$ in the last 5 years. Revisional procedures were performed for proximal enlargement in $26 \%$ or patients, port and tubing problems in $21 \%$ of patients, and erosion in $3.4 \%$ of patients. An additional systematic review of proximal pouch enlargement found a reoperation rate of approximately $5 \%$ over 2 years [18]. Erosion or migration of the band into the lumen of the stomach has an incidence of $1.4 \%$ as reported in a recent meta-analysis of almost 16,000 patients [19]. Another serious long-term complication unique to the gastric band is esophageal dilation and dysmotility, or "pseudo-achalasia." In a study by DeMaria et al. [20] up to $71 \%$ of patients who underwent placement of a gastric band had a significantly increased esophageal diameter on follow-up imaging studies. Of these patients, $72 \%$ had prominent symptoms of dysphagia, vomiting, or reflux, for which band explantation was recommended. Evidence has shown that the number of reoperations after gastric banding is increasing in the USA. In a retrospective outcome study, Spivak et al. [21•] observed a failure rate (defined as band explantation or less than $25 \%$ EWL) as high as $51.1 \%$ in 10 years. Moreover, the number of gastric-band-related reoperations increased from 579 in 2005 to 1,132 in 2008 (196\%) as observed by physicians from Duke University [22]. Furthermore, this study also found patients undergoing a gastric bypass procedure concomitant with a band-related reoperation were at increased risk of intraoperative and postoperative complications $[23 \cdot \bullet]$.

\section{Is the Laparoscopic Gastric Band a Good Procedure, and for Whom?}

With the problem of obesity growing, bariatric surgery remains the only durable treatment of weight loss for the morbidly obese patient. Since 1993, LABG has been a popular choice of procedure owing to low perioperative morbidity and relative ease of placement of the band. Research demonstrates that LAGB may provide significant reductions in weight loss and resolution of obesity-related comorbidities. However, LAGB is not the most effective surgical procedure to reduce weight. Current evidence, although predominantly observational, consistently demonstrates greater weight loss and improvements in obesityrelated conditions with RYGB compared with LABG [1]. The observed high rates of reoperation after LAGB due to late complications and failure has become a growing concern. The laparoscopic adjustable gastric band's decreased efficacy and higher risk for reoperation has caused some surgeons to prefer other bariatric procedures such as RYGB and more recently the vertical sleeve gastrectomy and question the role of LABG altogether.

Ultimately, disclosure of the efficacy and safety of LAGB should be discussed with all candidates wishing to undergo bariatric surgery. With lower early complication rates and shorter operative time and hospital length of stay, LAGB may be preferable for some patients [14]. However, late complications and risk of reoperation should also be discussed. LAGB surgery may be considered to have favorable attributes for patients with lower BMI, younger patients, and women planning families. Given the decreased operative time and lower complication rate, it may also be a preferred option in older and sicker patients when the risks of more complex surgery are greater and slower, more controlled weight loss is preferable [12]. As such, we conclude that LAGB is still an efficacious and beneficial option for select patient populations.

\section{Conclusions}

LABG is not the most effective surgical procedure to reduce weight. Current evidence demonstrates greater weight loss and improvements in obesity-related conditions with RYGB compared with LABG. The laparoscopic adjustable gastric band's decreased efficacy and higher risk for reoperation has caused some surgeons to prefer other bariatric procedures. However, with lower early complication rates and shorter operative time and hospital length of stay, LAGB may be preferable for patients with lower BMI as well as higher-risk patients. LAGB remains an efficacious and beneficial option for such select patient populations.

Disclosure Patrick Hartendorp and Dana A. Telem declare that they have no conflict of interest.

Compliance with Ethics Guidelines This article does not contain any studies with human or animal subjects performed by any of the authors.

\section{References}

Papers of particular interest, published recently, have been highlighted as:

- Of importance

•• Of major importance

1. Bessesen DH. Update on obesity. J Clin Endocrinol Metab. 2008;93(6):2027-34.

2. - Ludwig DS, Pollach HA. Obesity and the economy: from crisis to opportunity. JAMA. 2009;301(5):533. Interesting and timely article discussing obesity and its tax on healthcare. Highlighting 
another reason bariatric surgery is effective, regardless of procedure.

3. Tice, et al. Gastric banding or bypass? a systematic review comparing the two most popular bariatric procedures. Am J Med. 2008;121(10):885-93.

4. Scortino, et al. Morbid obesity. Current surgical therapy. 10th edn. 2011.

5. Wilkinson LH, Peloso OA. Gastrick (reservoir) reduction for morbid obesity. Arch Surg. 1981;116(5):602-5.

6. Steffen. The history and role of gastric banding. Surg Obes Relat Dis. 2008;4(3 Suppl).

7. Gastric Banding. U.S. Food and Drug Administration. http:// www.fda.gov/MedicalDevices/ProductsandMedicalProcedures/ ImplantsandProsthetics/GastricBanding/default.htm.

8. A band to band comparison. http://www.lapbandcentral.com/en/ about/band_to_band_comparison.

9. Buchwald H, Williams SE. Bariatric surgery worldwide 2003. Obes Surg. 2004;14:1157-64.

10. Lee C, Zieve D. Laparoscopic gastric banding. National Institutes of Health. http://www.nlm.nih.gov/medlineplus/ency/article/ 007388.htm.

11. Valle E, Luu MB, et al. Frequency of adjustments and weight loss after laparoscopic adjustable gastric banding. Obes Surg. 2012;22:1880-3.

12. Dixon JB, Straznicky NE, Lambert EA, Schlaigh MP, Lambert GW. Laparoscopic adjustable gastric banding and other devices for the management of obesity. Circulation. 2012;126:774-85.

13. • O'Brien P, MacDonald L, Anderson M, et al. Long-term outcomes after bariatric surgery: fifteen-year follow up of adjustable gastric banding and a systematic review of bariatric surgical literature. Ann Surg. 2013;257(1):87-94. Important longitudinal studies comparing outcomes long-term. As the true differences in safety and efficacy of band versus other procedures should include long-term data this is a very important study.

14. Chakravarty P, Mclaughlin E, Whittaker D, et al. Comparison of laparoscopic adjustable gastric banding (LAGB) with other bariatric procedures; a systematic review of randomized control trials. Surgeon. 2012;10:172-82.

15. Brancatisano A, Wahlroos B, Brancatisano R. Improvement in comorbid illness after placement of the Swedish Adjustable Gastric Band. Surg Obes Relat Dis. 2008;4(3 Suppl):S39-46.

16. Lundell L. Principles and results of bariatric surgery. Dig Dis. 2012;30:173-7.

17. Lee DY, Guend H, et al. Outcomes of laparoscopic Roux-en-Y gastric bypass versus laparoscopic adjustable gastric banding in adolescents. Obes Surg. 2012;22:1859-64.

18. Egberts K, Brown WA, O'Brien PE. Systematic review of erosion after laparoscopic adjustable gastric banding. Obes Surg. 2011;21:1272-9.

19. Lyass S, Cunneen SA, Hagiike M, Misra M, Burch M, Khalili TM, Furman G, Phillips EH. Device-related reoperations after laparoscopic adjustable gastric banding. Am Surg. 2005;71:738-43.

20. DeMaria EJ, Sugerman HJ, Meador JG, Doty JM, Kellum JM, Wolfe L, Szucs RA, Turner MA. High failure rate after laparoscopic adjustable silicone gastric banding for treatment of morbid obesity. Ann Surg. 2001;233(6):809-18.

21. - Spivak H, Abdelmelek M, Beltran O, et al. Long-term outcomes of laparoscopic adjustable gastric banding and laparoscopic Roux-en-Y gastric bypass in the United States. Surg Endosc. 2012;26:1909-19. Important longitudinal studies comparing outcomes long-term. As the differences in safety and efficacy for band versus other procedures should include long-term data this is a very important study.

22. Worni M, Ostbye T, Shah A, et al. High risks for adverse outcomes after gastric bypass surgery following failed gastric banding: a population-based trend analysis of the United States. Ann Surg. 2013;257(2):279-86.

23. • Maggard M, Shugarman L, Suttorp M, et al. Meta-analysis: surgical treatment of obesity. Ann Intern Med. 2005;142(7): 548-59. Important review comparing the outcomes of different obesity procedures. 